\title{
Needle aponeurotomy for Dupuytren contracture: Effectiveness of postoperative night extension splinting
}

\author{
Louisa Tam BHSc OT Reg(Ont), Yin-Yin Chung MHSc OT Reg(Ont)
}

L Tam, Y-Y Chung. Needle aponeurotomy for Dupuytren contracture: Effectiveness of postoperative night extension splinting. Plast Surg 2016;24(1):23-26.

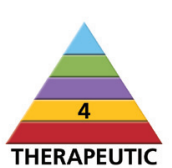

BACKGROUND: Night extension splinting has been used to treat patients with Dupuytren contracture to improve active range of motion (AROM) of the hand. A published case study demonstrated the benefit of splinting following needle aponeurotomy; however, no larger studies have evaluated the impact of postoperative splinting.

OBJECTIVES: To compare the impact of night extension splinting on AROM, specifically extension, following needle aponeurotomy for Dupuytren contracture.

METHODS: A retrospective chart review was conducted in which the charts of 53 patients who underwent needle aponeurotomy for Dupuytren contracture between 2009 and 2013 were reviewed. The control group consisted of patients who underwent needle aponeurotomy only, whereas the treatment group was also referred for fabrication of custom night extension splints after surgery. Comparisons in pre- and postoperative AROM measurements for the metacarpophalangeal (MCP) and proximal interphalangeal (PIP) joints were made between both groups of patients. The degrees of change in AROM for each joint were categorized in terms of levels of change: mild $\left(0^{\circ}\right.$ to $\left.29^{\circ}\right)$; moderate $\left(30^{\circ}\right.$ to $\left.60^{\circ}\right)$; and significant $\left(\geq 61^{\circ}\right)$.

RESULTS: All patients exhibited increased AROM after surgery for both $\mathrm{MCP}$ and PIP joints. Both groups had a greater increase in AROM in the MCP joint. Twelve joints from the control group had moderate changes and two from the treatment group had significant changes. When both groups were compared, the levels of change of AROM between both groups did not vary significantly.

CONCLUSION: Night extension splinting following needle aponeurotomy may not improve AROM of the MCP or PIP joints.

Key Words: Dupuytren contracture; Range of motion, articular; Retrospective studies; Splints; Therapy; Treatment outcome

T wo traditional and common surgical approaches for treating Dupuytren contracture are limited and complete palmar fasciectomies. Limited fasciectomy involves removal of the diseased tissue responsible for the contracture whereas a complete fasciectomy involves removal of the entire palmar fascia involved $(1,2)$. Needle aponeurotomy offers a less-invasive surgical approach to correct this deformity. This short-term solution involves transection of the digital and/or palmar fascial cord(s) with a small hypodermic needle to release the contracture (1-4).

Compared with limited fasciectomy, needle aponeurotomy has been found to be a good option for treating total extension deficits $\leq 90^{\circ}$ (5). Elderly patients with mild contractures prefer this surgical approach because it is associated with fewer postoperative complications and a more rapid recovery (6).

A randomized controlled trial from the United Kingdom compared patients who underwent hand therapy, with and without night extension splinting, following fasciectomy or dermofasciectomy. Active range of motion (AROM) for both patient groups was comparable. The study did not support the use of night extension splinting as a routine component of postoperative therapy (4). Another randomized controlled trial conducted in New Zealand yielded similar results.

\section{L'aponévrotomie à l'aiguille de la maladie de Dupuytren : l'efficacité d'une attelle d'extension nocturne postopératoire}

HISTORIQUE : L'attelle d'extension nocturne est utilisée pour améliorer l'amplitude active des mouvements (AAM) de la main des patients atteints de la maladie de Dupuytren. Une étude de cas publiée a démontré les avantages de l'attelle après une aponévrotomie à l'aiguille, mais aucune étude plus vaste n'a porté sur l'effet de l'attelle après l'operation.

OBJECTIFS : Comparer l'effet de l'attelle d'extension nocturne sur l'AAM, particulièrement l'extension, après une aponévrotomie à l'aiguille pour traiter la maladie de Dupuytren.

MÉTHODOLOGIE : Les chercheurs ont procédé à l'analyse rétrospective des dossiers de 53 patients qui avaient subi une aponévrotomie à l'aiguille pour traiter la maladie de Dupuytren entre 2009 et 2013. Le groupe témoin de patients avait subi seulement une aponévrotomie à l'aiguille, tandis que le groupe traité était également orienté vers la fabrication d'attelles d'extension nocturne adaptées après l'opération. Les chercheurs ont comparé les mesures de l'AAM des articulations métacarpophalangiennes (MCP) et interphalangiennes proximales (IPP) des deux groupes de patients avant et après l'opération. Ils ont classé le degré de changement de l'AAM de chaque articulation : léger $\left(0^{\circ}\right.$ à $\left.29^{\circ}\right)$; modéré $\left(30^{\circ}\right.$ à $\left.60^{\circ}\right)$ et important $\left(\geq 61^{\circ}\right)$.

RÉSULTATS : Tous les patients ont présenté une meilleure AAM des articulations MCP et IPP après l'opération. Les deux groupes présentaient une plus grande augmentation de l'AAM dans l'articulation MCP. Douze articulations du groupe témoin présentaient des changements modérés et deux articulations du groupe traité, des changements importants. Le taux de changement de l'AAM ne variait pas de manière significative entre les deux groupes

CONCLUSION : L'attelle d'extension nocturne après une aponévrotomie à l'aiguille n'améliore pas nécessairement l'AAM des articulations MCP ou IPP.

It concluded that hand therapy combined with postoperative night extension splinting may not be necessary unless there is considerable extension loss after surgery (7).

Although there is extensive published data regarding Dupuytren disease and its treatment options, there appears to be limited evidence regarding the effectiveness of night extension splinting as part of routine management following needle aponeurotomy. Because night extension splinting is a common component of therapy following fasciectomy to maintain digit extension, such a benefit may be extrapolated to postoperative needle aponeurotomy. The purpose of the present study was to explore the impact of custom night extension splinting on AROM following needle aponeurotomy by comparing patients who were referred for postoperative splinting with those who underwent the procedure alone.

\section{Setting}

\section{METHODS}

Trillium Health Partners (THP) is a full-service inpatient and outpatient community hospital, affiliated with the University of Toronto (Toronto, Ontario) Medical Program. The Hand Program is located in the ambulatory and outpatient services of the hospital. The program 


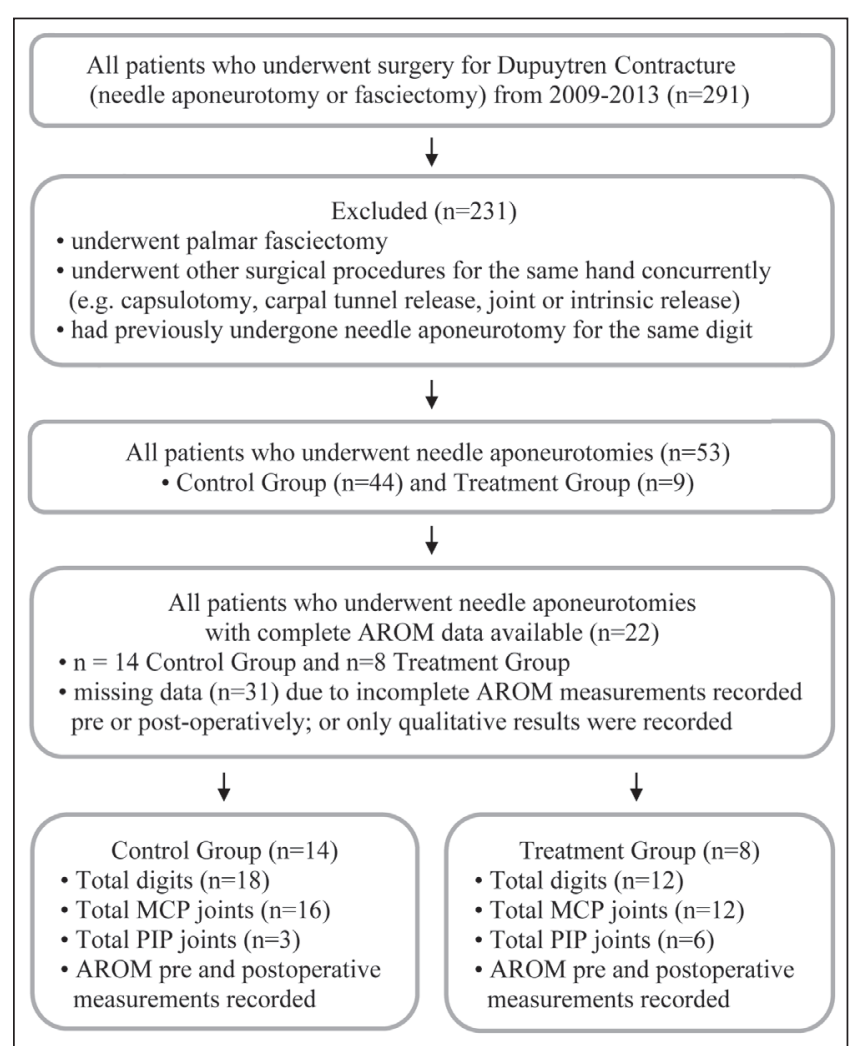

Figure 1) Data collection flowchart. AROM Active range of motion; MCP Metacarpophalangeal; PIP Proximal interphalangeal

provides treatment to patients who have hand and wrist conditions or injuries. Therapy is provided by occupational therapists (OTs), physiotherapists (PTs) and OT/PT assistants. The program accepts patients with referrals from specialists within THP (eg, plastic surgeons, orthopedic surgeons, neurologists) or non-THP-affiliated specialists with patients who reside within the catchment area of the hospital.

\section{Study design}

The present study was a retrospective chart review of patients who underwent needle aponeurotomy for Dupuytren contracture between 2009 and 2013, by a single plastic surgeon at THP in Toronto, Ontario. Only one plastic surgeon at THP administers needle aponeurotomy as a standard option for patients diagnosed with Dupuytren contracture. The AROM of affected joints at the time of the initial surgical consult and at the time of discharge were compared between the two groups, to explore the relationship between change in AROM and postoperative splinting. Before proceeding with the study, approval from THP's Research Ethics Board was received.

Patients in the control group underwent needle aponeurotomy and received a home exercise program, provided at the postoperative followup with the surgeon. This home exercise program consisted of AROM and passive range of motion exercises. These patients were not referred for custom extension splinting.

Patients in the treatment group were referred to the Hand Program at THP for custom extension splinting. These patients were treated by either OTs or PTs. A baseline AROM measurement of the affected digit(s) was documented by the therapist before the fabrication of a custom hand-based extension splint. Range of motion was measured using a finger goniometer placed dorsally over the joint in question, either the metacarpophalangeal (MCP) or proximal interphalangeal (PIP) joints. These hand-based splints were fabricated using a thermoplastic material that was custom molded to the patient's hand positioned in maximum extension. Patients were instructed to wear the splint at night only and were provided with a home exercise program
TABLE 1

\section{Demographic information of patients who underwent needle aponeurotomy, 2009 to $2013(n=53)$}

\begin{tabular}{lc}
\hline Age, years, mean (range) & 66 (41 to 81) \\
Sex & 47 \\
Male & 6 \\
Female & 40 \\
Hand dominance & 5 \\
Right & 7 \\
Left & 1 \\
Unknown & \\
Ambidextrous & 21 \\
Affected hand & 18 \\
Right & 12 \\
Left & 27 \\
Bilateral & D5 \\
Multiple digits affected & 8 (0 to 14) \\
Most affected digit & 51 (8 to 148) \\
Access (referral to therapy), days, mean (range) & 5.4 (1 to 19) \\
Length of stay in therapy, days, mean (range) & 56.3 (7 to 254) \\
Visits to therapy, n, mean (range) & \\
Duration of follow-up, days, mean (range) & \\
\hline
\end{tabular}

Data presented as $n$ unless otherwise indicated

consisting of AROM and passive range of motion exercises of all joints involved. The patients were instructed to complete the exercises, 10 repetitions per hour, during the day. When indicated, edema control, scar management and functional hand use were also addressed.

\section{Data collection}

Data extracted for the present study included: date of birth, sex, hand dominance, affected digit(s), operated digit(s), date of surgery, date of discharge from the surgeon and/or therapy, access time (referral to therapy), length of stay in therapy, number of visits to therapy, duration of follow-up (from date of initial consult to discharge), AROM, disciplines involved with treatment (OT, PT, OT/PT assistants) and compliance with splinting.

The present study's exclusion criteria included patients who: underwent other surgical procedures for the same hand concurrently; and had previously undergone needle aponeurotomy for the same digit. As the study progressed, additional patients were omitted due to: incomplete postoperative AROM data recorded; qualitative comments regarding AROM recorded as opposed to AROM measurements; or the inability to locate discharge notes from patient charts.

Data were extracted from patient charts from 2009 to 2013, originating from both the surgeon's office and from the health records department of THP. Data extraction from the surgeon's office was conducted onsite. A list of all patients treated for Dupuytren contracture was generated by the surgeon from the office database. Patient charts were subsequently retrieved and their data were entered into the research database.

For patients referred for custom extension splinting, a list of these patients were retrieved from the Hand Program's database on site. Patient charts dating more than one year after discharge from therapy were retrieved from the hospital's health records department off site. The above data were also entered into the research database.

Data from a total of 53 charts were entered into the database by both investigators of the present study. To ensure accuracy and complete collection of data, all were cross-referenced with the surgeon's office charts and THP's Meditech dictations for consultation, surgical and discharge reports. These were also cross-referenced with the Hand Program's therapy assessment and treatment notes in the patients' charts.

To calculate change in AROM after undergoing needle aponeurotomy for both the control and treatment groups, AROM must have been recorded at pre- and postoperative states. However, on completion of 
TABLE 2

Active range of motion data regarding the metacarpophalangeal (MCP) and proximal interphalangeal (PIP) joints pre- and postoperatively

\begin{tabular}{|c|c|c|c|c|c|c|}
\hline \multirow[b]{2}{*}{ Group } & \multicolumn{3}{|c|}{ MCP } & \multicolumn{3}{|c|}{ PIP } \\
\hline & Preoperative & Postoperative & Change & Preoperative & Postoperative & Change \\
\hline Control & $-35.00^{\circ}(n=16)$ & $-2.27^{\circ}$ & $32.72^{\circ}$ & $-76.67^{\circ}(n=3)$ & $-51.67^{\circ}$ & $25.00^{\circ}$ \\
\hline Treatment & $-42.92^{\circ}(n=12)$ & $-8.33^{\circ}$ & $34.58^{\circ}$ & $-54.17^{\circ}(n=6)$ & $-21.67^{\circ}$ & $32.50^{\circ}$ \\
\hline
\end{tabular}

' $n$ ' denotes number of joints

data entry, only 14 patients from the control group and eight from the treatment group had both pre- and postoperative AROM measurements (Figure 1).

Access time for patients to be admitted to the hand therapy program was determined from the date of the referral to the date of the initial assessment in therapy. The length of stay in therapy was determined from the date of the initial assessment in therapy to the date of discharge.

\section{Data analysis}

Comparative analysis regarding patient demographics (eg, age, sex, hand dominance, affected hand and digits); access time, length of stay and number of visits for the treatment group; overall and average change in AROM pre- and postoperatively between control and treatment groups; and duration of follow-up from the initial consultation with the surgeon to discharge was completed.

The changes in AROM between the control and treatment groups were categorized into three levels. The degrees of change in AROM for each joint were categorized in terms of levels of change: mild $\left(0^{\circ}\right.$ to $\left.29^{\circ}\right)$; moderate $\left(30^{\circ}\right.$ to $\left.60^{\circ}\right)$; and significant $\left(\geq 61^{\circ}\right)$.

\section{RESULTS}

Demographic information regarding the 53 patients in the present study are summarized in Table 1 . Approximately $40 \%$ of the patients were diagnosed with Dupuytren contracture in the right hand only, majority of which affected the fourth and fifth digits. Approximately $34 \%$ of patients were affected in the left hand only. The remainder of patients was affected bilaterally.

The duration of follow-up by the surgeon ranged from seven to 254 days, and averaged 48.9 days for the control group versus 100.4 days for the treatment group (from the date of initial consultation to discharge).

Sixty percent of the treatment group patients were treated by OTs and $30 \%$ by PTs. The remaining $10 \%$ were treated by both, which included the involvement of a therapy assistant. In one case, in which there was involvement of both professionals such that the PT was the primary therapist while the OT provided splinting assistance.

The degrees of AROM for both the control and treatment groups pre- and postoperatively for the MCP and PIP joints are summarized in Table 2. The change in AROM of these joints are also detailed (Table 2).

The levels of change in AROM for both groups in terms of mild, moderate and significant change (degrees) are summarized in Table 3. The majority of MCP joints demonstrated moderate levels of change in AROM for the control group and mild change for the treatment group (Table 3), whereas the majority of the PIP joints demonstrated mild levels of change for the control group and equally between mild to moderate levels for the treatment group (Table 3 ).

\section{DISCUSSION}

Our results do not strongly support the need for extension splinting following needle aponeurotomy. This implies that postoperative splinting may not be a necessary component of the treatment protocol. This is consistent with the findings of the studies from the United Kingdom and New Zealand mentioned previously. However, the decision to splint after the procedure may need to be evaluated by the surgeon on a case-by-case basis.

\section{TABLE 3}

Levels of change in active range of motion in selected joints between the control and treatment groups

\begin{tabular}{lccccccc}
\hline \multirow{2}{*}{ Change } & \multicolumn{3}{c}{ Control group $(\mathbf{n}=14)$} & & \multicolumn{3}{c}{ Treatment group $(\mathbf{n}=\mathbf{8})$} \\
\cline { 2 - 4 } \cline { 7 - 9 } & MCP & PIP & Total & & MCP & PIP & Total \\
\hline $0^{\circ}-29^{\circ}$ (mild) & 4 & 2 & 6 & & 6 & 3 & 9 \\
$30^{\circ}-60^{\circ}$ (moderate) & 12 & 1 & 13 & & 4 & 3 & 7 \\
$\geq 61^{\circ}$ (significant) & 0 & 0 & 0 & & 2 & 0 & 2 \\
\hline
\end{tabular}

MCP Metacarpophalangeal; PIP Proximal interphalangeal

As the data indicate, the patients in the present retrospective chart review appear consistent with literature describing the typical demographics of individuals diagnosed with Dupuytren contracture. The majority of our patients were male, average age was 66 years, and the fourth and fifth digits were the most commonly affected.

Both study groups, those who received home exercises and those who received night extension splinting in addition to the exercises, demonstrated between mild to moderate levels of change in MCP and PIP AROM postoperatively. Although the sample size of the present chart review was small, the data indicated no significant difference between the groups.

Study limitations for the present retrospective chart review include incomplete AROM data, AROM data being measured by different assessors (therapist versus surgeon), and treatment allocation bias. The study captured the entire population of patients who underwent needle aponeurotomy from 2009 to 2013; however, this still yielded a small sample size. Also, patient compliance with splinting was determined from patient self-report. Because splinting was the treatment being studied, if patient self-report was not reflective of splint use, then this would impact study outcomes.

Strategies to devise a stronger study include randomization of treatment allocation, larger sample size, standardized criteria for referral to therapy for splinting, and implementing an assessment protocol for initial and discharge visits with the surgeon.

From a health care resource perspective, the needle aponeurotomy alone can serve as an interim solution for long surgical waitlists and as a less-invasive option for those with mild forms of Dupuytren contracture. The procedure itself is quicker than a palmar fasciectomy and requires minimal use of surgical equipment and postoperative wound care. With this procedure, with or without splinting, the patient has the potential to gain AROM and perhaps avoid the need for more extensive surgery at a later time. Patients can also resume activities of daily living earlier after undergoing needle aponeurotomy because recovery time is shorter than other, more invasive, surgical procedures.

\section{CONCLUSION}

It appears that night extension splinting following needle aponeurotomy for Dupuytren contracture may not necessarily be more beneficial in producing more change in AROM postoperatively. The change in levels of AROM postoperatively did not vary greatly between control and treatment groups for both MCP and PIP joints.

ACKNOWLEDGEMENTS: The authors thank Dr Zvi Margaliot for his clinical expertise and Dr Morgan Lim for her research support that made this study possible. 


\section{REFERENCES}

1. Friedman R. Dupuytren's disease. Selected Readings in Plastic Surgery 1995;7:1-36.

2. Ball C, Pratt AL, Nanchahal J. Optimal functional outcome measures for assessing treatment for Dupuytren's disease: A systematic review and recommendations for future practice. BMC Musculoskelet Disord 2013; 14: 131.

3. Srinivasan RC, Shah AS, Jebson PJL. New treatment options for Dupuytren's surgery: Collagenase and percutaneous aponeurotomy. J Hand Surg 2010; 35A:1362-4.

4. Jerosch-Herold C, Shepstone L, Chojnowski AJ, Larson D, Barrett E, Vaughan SP. Night-time splinting after fasciectomy or dermofasciectomy for Dupuytren's contracture: A pragmatic, multi-centre, randomised controlled trial. BMC Musculoskelet Disord 2011;12:136.
5. van Rijssen AL, Gerbrandy FS, Ter Linden H, Klip H, Werker PM. A comparison of the direct outcomes of percutaneous needle fasciotomy and limited fasciectomy for Dupuytren's disease: A 6-week follow-up study. J Hand Surg 2006;31A:717-25.

6. van Rijssen AL, Ter Linden H, Werker PM. Five-year results of a randomized clinical trial on treatment in Dupuytren's disease: Percutaneous needle fasciotomy versus limited fasciectomy. Plast Reconstr Surg 2012;129:469-77.

7. Collis J, Collocott S, Hing W, Kelly E. The effect of night extension orthoses following surgical release of Dupuytren's contracture: A single-center, randomized, controlled trial. J Hand Surg 2013;38A:1285-1294. 